\title{
Monolithically Integrated Selectable Repetition-Rate Laser Diode Source of Picosecond Optical Pulses
}

\author{
Xuhan Guo*, Vojtech Olle, Adrian Quarterman, Adrian Wonfor, Richard Penty and Ian White \\ Centre for Photonic Systems, Electrical Engineering Division, Engineering Department, University of Cambridge, \\ 9, JJ Thomson Avenue, Cambridge CB3 0FA, UK \\ *Corresponding author:xg218@cam.ac.uk
}

Received Month X, XXXX; revised Month X, XXXX; accepted Month X,

XXXX; posted Month X, XXXX (Doc. ID XXXXX); published Month X, XXXX

We describe the characterization of a monolithically integrated photonic device for short pulse generation featuring a modelocked laser diode, a Mach Zehnder modulator, and a semiconductor optical amplifier (SOA). The integrated device is designed for fabrication by a generic foundry scheme with a view to ease of design, testing and manufacture. Trains of $6.8 \mathrm{ps}$ pulses are generated at repetition rates which are electronically switchable from $14 \mathrm{GHz}$ to $109 \mathrm{MHz}$. The SOA boosts the peak power by $7.4 \mathrm{~dB}$, and the pulses are compressible to 2.4 ps by dispersion compensation using single-mode telecommunications fibre. (c) 2013 Optical Society of America

OCIS Codes: (140.4050) Mode-locked lasers, (130.3120) Integrated optics devices, (250.4110) Modulators, (250.5980) Semiconductor optical amplifiers

http://dx.doi.org/10.1364/OL.99.099999

Ultrafast optical pulse sources have undergone extensive research and commercial development, having found many applications in fields as diverse as optical communications [1], high speed sampling [2], clock distribution [3], and fiber radio [4]. While traditional solidstate and fibre-based sources are generally capable of the highest performance, monolithically mode-locked laser diodes (MMLLDs) are potentially a commercially attractive alternative owing to their properties which include low cost, small footprint, direct electrical pumping, and high wallplug efficiency. While being promising sources in these respects, they have largely been unable to achieve the performance required for the applications described above, in general having low peak powers, long, highly chirped pulses, and high repetition rates. In order for MMLLDs to compete with established solid-state and fibre laser systems it is necessary to improve the performance of currently available devices.

The high repetition rates of most MMLLDs are caused by their inherently short cavities, and the low peak powers are due both to the high repetition rates, and to the low driving currents required for stable mode-locked operation [5]. The repetition rate issue has been addressed monolithically, for example by Cheung et al. [6] who have demonstrated the emission of $36 \mathrm{ps}$ pulses at 1 $\mathrm{GHz}$ from a $41 \mathrm{~mm}$ long device, and $\mathrm{Li}$ et al. [7] who have demonstrated passive modelocking at a fundamental repetition rate of $2.1 \mathrm{GHz}$ in a $20 \mathrm{~mm}$ long monolithic twosection quantum dot laser. The peak powers and repetition rates of these low repetition rate lasers are ultimately limited by the effects of large material losses, nanosecond gain recovery times, and high dispersion, all of which become more problematic as cavity lengths increase. Increased peak powers however can be achieved by using monolithic master oscillator power amplifier
(MOPA) configurations. Olle et al. [8] have demonstrated 10 ps Fourier transform limited pulses with peak powers exceeding $0.31 \mathrm{~W}$ at a $20.4 \mathrm{GHz}$ repetition rate. Faugeron et al [9] have reported $10 \mathrm{GHz}$ subpicosecond pulses with $18 \mathrm{~W}$ peak power from an asymmetrical cladding singlesection quantum-dash mode-locked laser. Ahmad et al [10] also reported fundamental $(\sim 5.2 \mathrm{GHz})$ and subharmonic hybrid modelocking of a high power $(220 \mathrm{~mW})$ monolithic semiconductor laser.

Non-monolithic MOPA configurations can achieve high peak powers and low repetition rates at the same time, but only with increased cost and complexity compared with monolithic devices. For example, Schlauch et al. [11] have presented a train of $622 \mathrm{fs}$ pulses with a record high peak power of $2.5 \mathrm{~kW}$, repetition rate of 330 $\mathrm{MHz}$ from a distributed passively mode locked MOPA device followed by an optical isolator, a tapered amplifier and a linear pulse compressor. Ding et al. [12] have reported picosecond optical pulses with power of 208.2 $\mathrm{mW}$, pulse energy of $321 \mathrm{pJ}$, and peak power of $30.3 \mathrm{~W}$ at $648 \mathrm{MHz}$ from a quantum-dot mode-locked laser diode and an external tapered quantum-dot semiconductor optical amplifier. To the best of our knowledge, no monolithically integrated source has achieved both high peak power and variable low repetition rate simultaneously, though similar configurations to the one described in this paper have been demonstrated in monolithic formats for applications in communications [13], but not as a route to high peak powers and low repetition rates.

At present, although photonic integrated circuits (PIC) have shown promise as a transformative technology, their widespread adoption has been hindered by the prohibitive cost of designing bespoke components and accessing foundry facilities. One proposed solution would be for 
PICs to take a similar development path to that followed by microelectronic integration technology [14], that is, to provide a basic set of standardized components using generic from which more complex circuits can be built. Recently however InP generic foundries have used a selection of basic optoelectronic components to assemble Application Specific Photonic Integrated Circuits (ASPICs) [15], in our work therefore, particularly given our need for repeated device optimization, this generic foundry design and fabrication scheme is used.

In this paper we present a novel scheme where a MLLD, Mach-Zehnder Modulator (MZM) and SOA are monolithically integrated using an InP foundry. The MLLD is used to generate a stable high repetition rate train of low peak power pulses which is then modulated by the MZM, thus decreasing the repetition rate of the pulse train and providing repetition rate flexibility. Finally, the SOA boosts the power particularly of the low repetition rate pulse train, thus resulting in a more efficient conversion of average power to peak power.

As well as improved peak powers and variable repetition rates, a further advantage of this design is that it enables a single device to output pulse trains covering a very wide parameter space, allowing the device to be optimized electronically to suit the performance requirements of a given application. This is a particularly advantageous feature for applications where optimization of the source can yield significant improvements in the system performance, such as in multi-photon imaging [16], optical coherence tomography [17], and optical sampling [18].

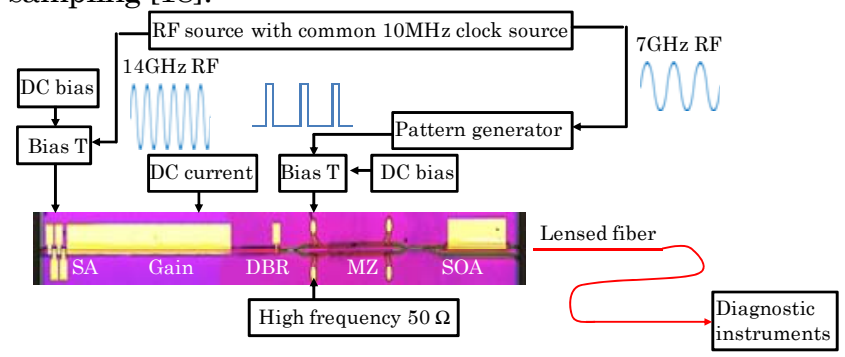

Fig. 1. A microscope image of the integrated device and a schematic diagram of the drive electronics used for hybrid modelocking, modulation and amplification of the pulse train.

Fig. 1 shows a microscope image of the device and a schematic of the associated experimental electronics. The indium phosphide based multi quantum-well device studied in this work is fabricated using a photonic integrated circuit generic foundry approach [14]. The laser cavity consists of an HR coating on one facet, four $50 \mu \mathrm{m}$ long absorbers, a $2.1 \mathrm{~mm}$ long gain section, and $0.57 \mathrm{~mm}$ of passive waveguide with a DBR section as an output coupler. The DBR has a coupling strength of $50 \mathrm{~cm}^{-1}$, and a length of $50 \mu \mathrm{m}$. The four pads of the saturable absorber can be independently electrically connected, allowing the length of the absorber to be varied to optimize the laser performance. An absorber length of $100 \mu \mathrm{m}$ is used throughout this work. The total laser cavity length is 3.0 mm resulting in a fundamental repetition rate of $14 \mathrm{GHz}$. Hybrid modelocking is achieved with the gain section driven by a DC current and the absorber section driven by a reverse $\mathrm{DC}$ voltage plus $\mathrm{RF}$ modulation. The device is mounted on a copper heaksink whose temperature is maintained at $20^{\circ} \mathrm{C}$, leading to a lasing wavelength of $1540 \mathrm{~nm}$.

The pulse train emitted by the laser is split by a $1 \times 2$ MMI coupler into the Mach-Zehnder modulator. Each arm of the MZM contains one $1090 \mu \mathrm{m}$ long electro-optic phase modulator. The modulator in one arm of the MZM is driven via a bias tee with the $\mathrm{DC}+\mathrm{RF}$ voltage required for inducing a phase shift alternating between 0 and $\Pi$ or $\Pi$ and $2 \Pi$ so as to alternately cause constructive or destructive interference with the other arm, and therefore to modulate the signal emitted from the MZM as a whole. The reduced repetition rate pulse trains are directed either to a passive waveguide output channel, or to an output waveguide containing a $750 \mu \mathrm{m}$ long SOA. Both outputs are AR coated and angled at 7 degrees to minimize feedback. Pulses from either output are collected by a lensed fiber for transmission to the diagnostic instruments. The external RF signal sources for hybrid modelocking and MZM modulation are synchronized through a common $10 \mathrm{MHz}$ clock. A programmable pattern generator is then used to generate the desired drive waveform to achieve a specific repetition rate. By applying a waveform of variable frequency and duty cycle to one arm of the MZM, the $14 \mathrm{GHz}$ pulse train from the MLLD is then modulated and variable repetition rates can be generated. Finally, the power of the modified repetition rate pulse trains is amplified by the SOA section which is driven by a DC current.

The stable mode-locking regime lies in the gain section current range of 160-190 mA and absorber section reverse bias voltage of 1.7-2.1 V. Optimal hybrid modelocking is demonstrated by applying a $180 \mathrm{~mA}$ DC current to the gain section (2.4 times higher than the threshold current) and $-2.04 \mathrm{~V}$ DC bias voltage with $\mathrm{RF}$ modulation to the saturable absorber section via a bias tee. The $R F$ modulation frequency is chosen to be close to the fundamental repetition rate of $13.94 \mathrm{GHz}$.
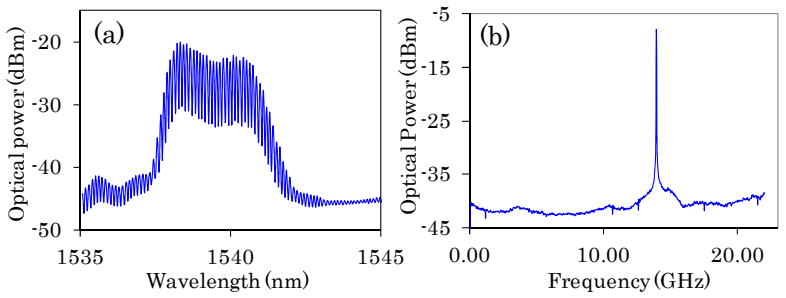

Fig. 2. (a) Optical spectrum with a centre wavelength of $1540 \mathrm{~nm}$ and a spectral width of $2.54 \mathrm{~nm}$ (b) RF spectrum of the laser output, with a peak frequency of $13.94 \mathrm{GHz}$.

Fig. 2 and Fig. 3 show the optical spectrum, RF spectrum and autocorrelations of a typical pulse train. The measured spectral bandwidth in this case is $2.54 \mathrm{~nm}$ and the pulse duration $7.8 \mathrm{ps}$ assuming a $\operatorname{sech}^{2}$ pulse 
profile, so that the pulse is 8 times transform limited, and hence is strongly chirped. The chirp may result from the changes in refractive index caused by the change of carrier density in the absorber and the gain sections, and also from waveguide dispersion.
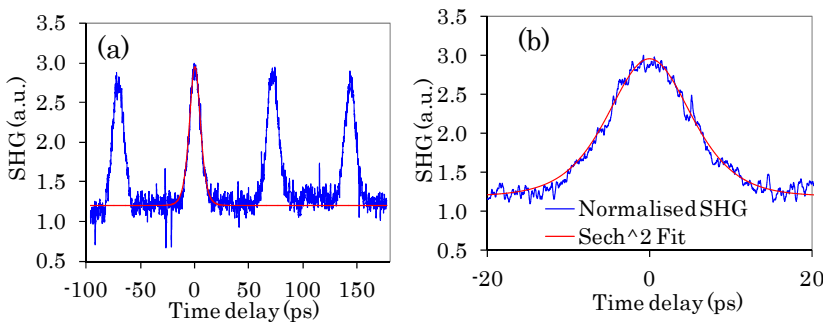

Fig. 3. Colinear autocorrelations of the pulse train emitted by the hybrid mode-locked MLLD, showing (a) a train of peaks of equal heights, and (b) a sech-squared fit to the zero delay peak corresponding to a pulse duration of $7.8 \mathrm{ps}$.

Pulses with shorter durations are achieved by dispersion compensation of the laser output which further increases the peak power while maintaining the pulse quality. For the optimal pulse compression and under similar driving conditions to those above, the investigated pulse is chosen with a spectral width of $1.76 \mathrm{~nm}$ and initial pulse duration of $8.7 \mathrm{ps}$. The time-bandwidth product of 1.93 is 6.1 times larger than the transformlimited value of 0.315 for $\operatorname{sech}^{2}$ pulses.
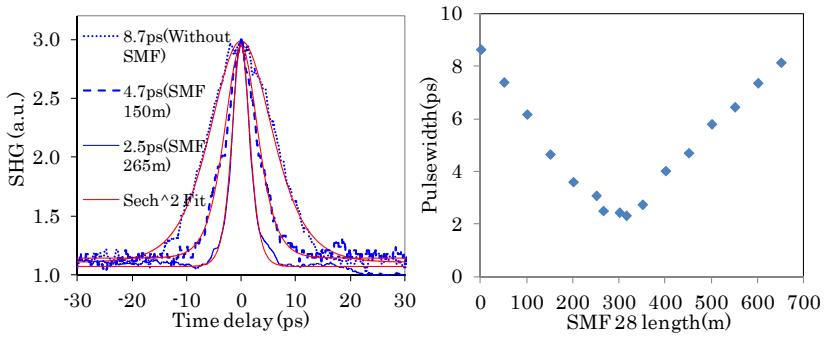

Fig. 4. Colinear autocorrelation traces after dispersion compensation in different lengths of SMF-28 fiber. The shortest measured pulse width is $2.4 \mathrm{ps}$ at a fibre length of $325 \mathrm{~m}$.

Different lengths of standard SMF-28 fibre, with a group velocity dispersion of $16 \mathrm{ps} / \mathrm{km} / \mathrm{nm}$ at $1550 \mathrm{~nm}$, have been used to compensate for the linear component of the dispersion. The length of the single-mode fiber is optimized for shortest pulse width, and the autocorrelation traces measured after various lengths of the SMF-28 are shown in Fig. 4. The shortest dispersion compensated pulse, $2.4 \mathrm{ps}$ in duration, has been achieved with a fibre length of $315 \mathrm{~m}$, corresponding to a total dispersion of $5.0 \mathrm{ps} / \mathrm{nm}$. The reduction in pulse duration corresponds to a reduction from 6.1 to 1.7 times transform limited. A small pedestal can be seen in the autocorrelation of this pulse, which is indicative of the presence of high-order dispersion which is not compensated for in the SMF-28 fiber, but could be compensated with more complex experimental approaches such as grating pairs [19], but clearly the majority of energy is in the main pulse.
Modulation of the pulse train has been achieved by applying square wave modulation signals between $4.3 \mathrm{~V}$ and $2.3 \mathrm{~V}$ at the desired frequency and duty cycle to one arm of the MZM. Extra peaks occur in the RF spectra corresponding to the modulation frequencies. Fig. 5 shows oscilloscope traces of an unmodulated $14 \mathrm{GHz}$ pulse train and the same pulse train modulated to a decreased repetition rate of $109 \mathrm{MHz}$ measured via the passive waveguide output. The inset of Fig. 5 (b) shows that two pulses are transmitted by the MZM rather than a single pulse. This is because the bandwidth of the pattern generator $(12.5 \mathrm{GHz})$ is lower than the $14 \mathrm{GHz}$ fundamental repetition rate of the laser. A higher bandwidth pattern generator would allow single pulse picking.

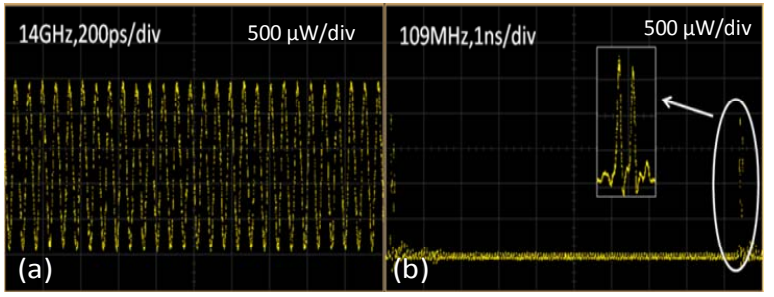

Fig. 5. Sampling oscilloscope traces of (a) an unmodulated pulse train at a repetition rate of $14 \mathrm{GHz}$ (200 ps/div), (b) a modulated pulse train at $109 \mathrm{MHz}$ (1 ns/div).

One of the merits of using a MZM to set the repetition rate is that the output pulse train can be tuned electronically in discrete steps over a large range, with tuning by a factor of 128 being demonstrated in this paper. In addition to this discrete step tuning, hybrid modelocking also allows some continuous tuning of the repetition rate by changing the frequency of the $R F$ voltage applied to the saturable absorber. The fundamental repetition rate measured can be continuously tuned over a range of $20 \mathrm{MHz}$ without loss of stability. This combination of coarse discrete switching and fine continuous control of the repetition rate gives the device a large degree of repetition rate flexibility and accuracy. This flexibility makes devices of this type potentially suitable as a low cost source for applications such as fluorescence imaging [16] and Asynchronous Optical Sampling (ASOPS) [18].

Pulse trains from the MZM pass through a $2 \times 2$ MMI coupler, from where they are sent either to a direct output via a passive waveguide, or to an output via an SOA. Experimentally, the SOA section transparency current is measured to be $12.5 \mathrm{~mA}$ using the method described in [20]. It is found that if high currents are applied to the SOA the hybrid mode-locking is therefore destabilized due to amplified feedback and noise caused by the small reflections from the butt joints in the device Modelocking stability is maintained by limiting the current applied to the SOA, which in turn limits the amplification which could be achieved. Autocorrelations of the pulse train after modulation and amplification are shown in Fig. 6. The measured pulse width of $6.8 \mathrm{ps}$ is slightly shorter than 
that of the pulse without modulation and amplification, which may be due to dispersion compensation by the small positive dispersion introduced in the phase modulators. While autocorrelations of the unmodulated pulse train show a sequence of equal-height pulses, the three dominant peaks of unequal heights in Fig. 6 (a) are consistent with the autocorrelation of a pair of isolated pulses rather than a continuous pulse train. The small peak after the three dominant peaks is due to the imperfect extinction ratio in the MZM, and could be reduced by improved fabrication.
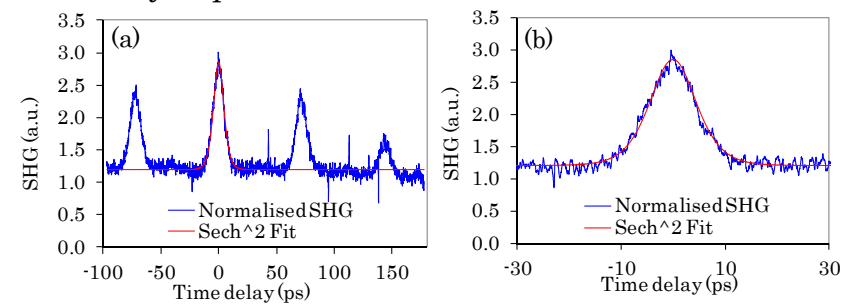

Fig. 6. Collinear autocorrelations of a modulated and amplified pulse train. (a) group of peaks and (b) zero delay peak.

The measured output parameters for (1) pure hybrid modelocking and (2) hybrid modelocking plus modulation to a repetition rate of $109 \mathrm{MHz}$ and SOA amplification at a current of $31 \mathrm{~mA}$, are compared in Table 1.

Table 1 Parameters of Pulses Output by (1) the MLLD Alone and by (2) the MLLD-Modulator-MOPA Source

\begin{tabular}{lcc}
\hline \multicolumn{1}{c}{ Pulse performance } & $(1)$ & $(2)$ \\
\hline Spectra width (nm) & 2.54 & 2.11 \\
Pulse width (ps) & 7.78 & 6.81 \\
Time bandwidth product & 2.50 & 1.82 \\
Max. pulse energy (pJ) & 0.10 & 0.41 \\
Fiber coupled average power (mW) & 1.20 & 0.45 \\
Max. peak power (mW) & 9.57 & 53 \\
Min. rep rate (GHz) & 14 & 0.11 \\
\hline \hline
\end{tabular}

In summary, a monolithically integrated short pulse source consisting of an MLLD, a MZM and a MOPA is demonstrated. The MZM and MOPA expand the functionality of the device beyond that of the MLLD alone, enabling increased peak powers and tunable repetition rates. The design and fabrication are achieved using a generic foundry scheme. 6.8 ps pulses are generated at repetition rates which can be switched electronically from $14 \mathrm{GHz}$ to $109 \mathrm{MHz}$. Pulse compression to a duration of $2.4 \mathrm{ps}$ in SMF-28 fibre is also demonstrated, increasing the peak power by a factor of three. Also, even with a low current applied to the SOA, the pulse peak power is increased by $7.4 \mathrm{~dB}$ without major degradation of any of the pulse parameters. "This proof-of-principle device has shown the interplay of pulse picking and amplification. The use of pulse picking to enable boosted peak powers after amplification makes possible performance levels which are approaching those that would be suitable for biophotonic applications, despite using a compact and cost effective platform.

This research leading to these results has received support from the UK EPSRC COPOS award
EP/H022384/1 and from the European Commission's Seventh Framework Programme FP7/2007-2013 under grant agreement NMP 228839 EuroPIC.

\section{Reference}

1. R. Kaiser and B. Huttl, IEEE J. Sel. Topics Quantum Electron., 13, 125-135 (2007).

2. K. J. Weingarten, M. J. W. Rodwel, and D. M. Bloom, IEEE J. Quantum Electron., 24, 198-220 (1988).

3. P. J. Delfyett, D. H. Hartman, and S. Z. Ahmad, J. Lightw. Technol., 9, 1646-1649 (1991).

4. A. J. C. Vieira, P. R. Herczfeld, A. Rosen, M. Ermold, E. E. Funk, W. D. Jemison, and K. J. Williams, IEEE Trans. Microw. Theory Techn., 49, 1882-1887 (2001).

5. P. P. Vasil'ev, Ultrafast diode lasers: Fundamentals and Applications (Artech House, Incorporated., 1995).

6. S. Cheung, B. Jong-Hwa, R. P. Scott, N. K. Fontaine, F. M. Soares, Z. Xiaoping, D. M. Baney, and S. J. Ben Yoo, IEEE Photon. Technol. Lett., 22, 1793-1795 (2010).

7. Y. Li, M. Breivik, F. Cheng-Yong, B. O. Fimland, and L. F. Lester, IEEE Photon. Technol. Lett., 23, 1019-1021 (2011).

8. V. F. Olle, M. G. Thompson, K. A. Williams, R. V. Penty, and I. H. White, OFC, 1-3 (2009).

9. M. Faugeron, F. Lelarge, M. Tran, Y. Robert, E. Vinet, A. Enard, J. Jacquet, and F. Van Dijk, Selected Topics in Quantum Electronics, IEEE Journal of 19, 1101008-1101008 (2013).

10. F. R. Ahmad and F. Rana, IEEE Photon. Technol. Lett., 20, 1308-1310 (2008).

11. T. Schlauch, M. Li, M. R. Hofmann, A. Klehr, G. Erbert, and G. Trankle, Electron. Lett., 44, 678-679 (2008).

12. Y. Ding, R. Aviles-Espinosa, M. A. Cataluna, D. Nikitichev, M. Ruiz, M. Tran, Y. Robert, A. Kapsalis, H. Simos, C. Mesaritakis, T. Xu, P. Bardella, M. Rossetti, I. Krestnikov, D. Livshits, I. Montrosset, D. Syvridis, M. Krakowski, P. LozaAlvarez, and E. Rafailov, Opt. Express., 20, 14308-14320 (2012).

13. B. R. Koch, J. S. Barton, M. Masanovic, Z. Hu, J. E. Bowers, and D. J. Blumenthal, IEEE Photon. Technol. Lett., 19, 641643 (2007).

14. P. K. Tien, Rev. Mod. Phys. 49, 361-420 (1977).

15. M. Smit, X. Leijtens, E. Bente, J. van der Tol, H. Ambrosius, D. Robbins, M. Wale, N. Grote, and M. Schell, Optoelectronics, IET 5, 187-194 (2011).

16. M. Kuramoto, N. Kitajima, H. Guo, Y. Furushima, M. Ikeda, and H. Yokoyama, Opt. Lett., 32, 2726-2728 (2007).

17. U. Sharma, E. W. Chang, and S. H. Yun, Opt. Express., 16, 19712-19723 (2008).

18. P. A. Elzinga, R. J. Kneisler, F. E. Lytle, Y. Jiang, G. B. King, and N. M. Laurendeau, Appl. Opt., 26, 4303-4309 (1987).

19. M. Stern, J. P. Heritage, and E. W. Chase, IEEE J. Quantum Electron., 28, 2742-2748 (1992).

20. Y. H. Kao, T. J. Xia, M. N. Islam, and G. Raybon, J. Appl. Phys., 86, 4740-4747 (1999). 